Ik Yang · Curtis W. Hayes · Jon A. Jacobson

David A. Jamadar · Stewart Wang

\title{
Unique foreign body injury: bamboo penetration of thigh and pelvis while skiing
}

Received: 16 April 2002 / Accepted: 14 June 2002 / Published online: 24 August 2002

(c) ASER 2002

\begin{abstract}
We present a case of traumatic bamboo foreign body penetration through the posterior thigh extending cephalad into the pelvis sustained during skiing. The unsuspected bamboo foreign body was missed prospectively on the initial portable trauma radiograph of the pelvis, but was retrospectively quite apparent as a linear $12 \times 2-\mathrm{cm}$ radiolucent region overlying the left pelvis and hip region. On CT examination, the bamboo stick appeared as a round cylindrical air-filled structure of high density compared to soft tissue - the typical appearance of bamboo. Bamboo foreign bodies can be recognized radiographically by this typical appearance, since it is one of the few wood products that causes high attenuation relative to soft tissue on CT.
\end{abstract}

\section{Keywords Trauma $\cdot$ Foreign body $\cdot$ Bamboo $\cdot$ CT}

Foreign objects in the body are frequent causes of surgical exploration, and diagnosis of the presence is often difficult if they are initially unsuspected from clinical examination. This is particularly true of wooden and other nonradiopaque materials.

We recently encountered a foreign body injury in which a bamboo stick penetrated from the posterior

I. Yang $\cdot$ C.W. Hayes $(\bowtie) \cdot$ J.A. Jacobson $\cdot$ D.A. Jamadar

Department of Radiology,

University of Michigan Health Systems,

Ann Arbor, Michigan, USA

E-mail: hayescw@umich.edu

Tel.: + 1-734-6473037

Fax: + 1-734-9369723

S. Wang

Department of Surgery,

University of Michigan Health Systems,

Ann Arbor, Michigan, USA

C.W. Hayes

Department of Radiology,

University of Michigan Health Systems, Taubman Center,

Room 2910A , 1500 East Medical Center Drive,

Ann Arbor, MI 48109-0326, USA thigh into the pelvic cavity. The correct diagnosis and most efficient surgical approach was aided with computed tomography (CT) of the pelvis.

\section{Case report}

A 22-year-old man without significant past medical history presented with severe left hip pain after a fall skiing. Apparently he had fallen at a fairly high speed, landing on his left buttock, and felt immediate sharp, shooting pain. He initially believed he had landed on a rock, but upon further questioning thought it possible that he might have struck a bamboo pole marking the edge of the ski trail. He had not been able to bear weight, but had no numbness or tingling sensations.

On physical examination, he showed voluntary guarding during his abdominal exam. The left side of his abdomen was diffusely tender. On the proximal posterior aspect of his left thigh there was a small $1.5-\mathrm{cm}$ puncture wound (Fig. 1). There was no active bleeding. Small fragments of foreign body material were visualized at the wound site, but the type and extent of the penetrating injury was unclear.

A portable AP pelvis radiograph obtained with the patient on a backboard (Fig. 2) showed no evidence of fracture or dislocations. A small amount of subcutaneous air tracking to the hip joint was noted. A long, rectangular area of gas radiolucency projected over the left upper thigh, hip, and left pelvis (Fig. 2) which prospectively was thought to be a backboard artifact.

On the suspicion that a more extensive penetrating injury had occurred than first seemed apparent, an abdominal and pelvic CT scan was obtained (Fig. 3). A cylindrical, hollow foreign body measuring $1.5 \mathrm{~cm} \times 1.5 \mathrm{~cm} \times 13 \mathrm{~cm}$ was identified extending from the left iliopsoas muscle adjacent to the left hip, cephalad through the iliacus muscle, into the left pelvis. The left descending colon was slightly displaced medially by the object, but there was no free intraperitoneal air or fluid to suggest actual bowel wall penetration. The foreign body showed a thin, highdensity rim with central air. The surrounding muscle showed slightly low-attenuation edema. A small amount of gas was noted in the left hip joint, indicating penetration of the joint. Caudal to the foreign body, there was gas tracking within the muscles of the left posterior thigh leading to an abrasion at the posterior aspect of the proximal left thigh, at the point of the penetrating injury.

In the operating room, an anterior incision was made directly over the cranialmost end of the stick. A 13-cm length of bamboo wrapped in tape was extracted through the incision as a single piece (Fig. 4). The wound was thoroughly irrigated, a drain placed, and the patient was put on antibiotics. In spite of the proximity of the 


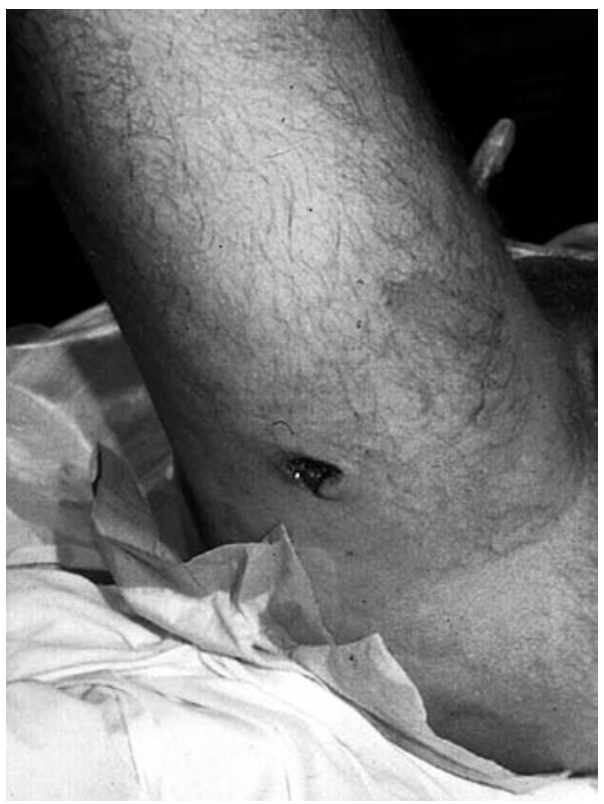

Fig. 1. Photograph of left thigh showing a small puncture wound at the posterolateral aspect of the upper thigh

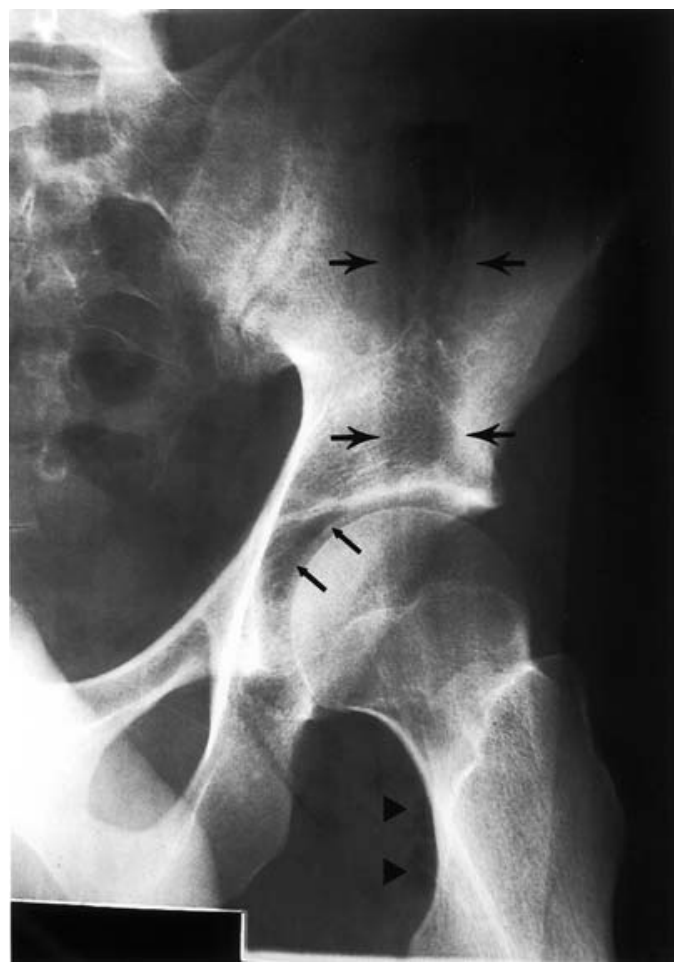

Fig. 2. Pelvic AP radiograph shows a small amount of subcutaneous air around the hip joint (arrowheads); the medial cartilage of the femoral head is outlined by air (small arrows), indicating definite gas within the joint. A long tubular radiolucent region (arrows) can also be seen, representing gas within the hollow bamboo stick. Prospectively, this was initially thought to represent artifact from the backboard

bamboo stick to the descending colon (Fig. 3a), there was no injury to the bowel or any major vessels. After 5 days, the patient was discharged.

\section{Discussion}

Most foreign bodies are introduced by penetrating injuries of the body. Metallic foreign bodies are easily detected on simple radiography, but the detection of wooden materials is difficult as they are usually either less dense or isodense to overlying soft tissue [1].

CT has inherently better contrast resolution than plain radiography. Published experience with the imaging of foreign bodies with CT includes in vitro studies [2] and occurrences in the orbit $[3,4,5,6,7]$, tracheobronchial tree [8], abdomen [9, 10], lung [11], and extremity [12]. Ultrasonography has also proven useful in the identification of nonradiopaque foreign bodies, especially when located superficially [13].

An in vitro comparison of $\mathrm{CT}$, xeroradiography, and radiography in the detection of soft tissue foreign bodies (toothpick) showed that neither a soaked nor an unsoaked toothpick could be definitely identified by xeroradiography or mammographic radiography in the muscle of the extremity. CT visualized both toothpicks, although the soaked toothpick was of the same density as clotted blood in adjacent blood vessels [2].

CT attenuation values of wooden foreign bodies in the extremity are reported to vary from low to high according to the length of time the wooden particle is in the body [12].

Furthermore, superimposed inflammatory changes probably affect the $\mathrm{CT}$ densities of wooden foreign bodies, and those retained for longer periods of time may even become calcified [2, 8, 12].

Nakata et al. [11] reviewed CT features of two cases of post-traumatic bamboo foreign bodies in lung parenchyma. Chest radiography showed a linear opacity in the left upper lobe and a large cystic opacity with airfluid level in the left lower lobe. The CT findings were of relatively cylindrical structures of high density and were highly suggestive of bamboo. The authors further evaluated the density characteristics of bamboo by scanning a piece of fresh bamboo in a water bath. This confirmed the high density of the bamboo, with a CT attenuation value ranging from 120-180 HU. Unlike most wooden foreign bodies, bamboo appears to maintain its original cylindrical structure when in the body for many years.

There have been several reports of CT of intraorbital wooden foreign bodies [3, 4, 5, 6, 7]. Because of its lower electron density, wood shows low X-ray attenuation. In vitro, most types of wood are less dense than fat [7], and wooden orbital foreign bodies in the acute stage are less dense than orbital fat [2]. In some cases, wooden fragments were initially misinterpreted as air bubbles on CT. Older intraorbital wooden foreign bodies are reportedly isodense with or denser than soft tissue [6]. Presumably this is caused by "soaking" or via the inflammatory process, converting "dry" wood to "wet" within a few days, resulting in dramatic increases in attenuation on CT [3].

Roberts and Leehey [7] reported intraorbital foreign body mimicking air at CT. CT revealed a $2-\mathrm{cm}$ linear 

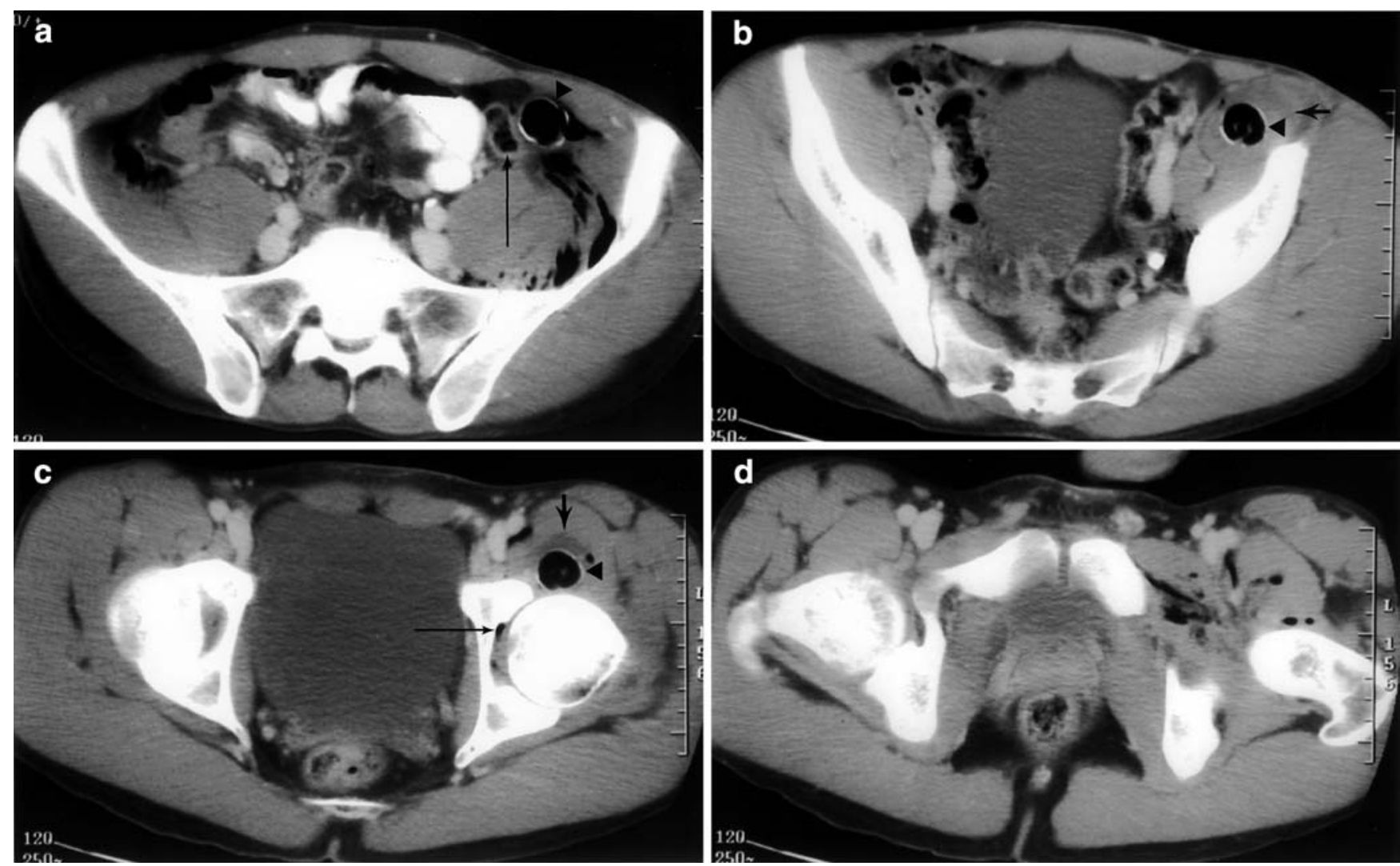

Fig. 3a-d. CT scan of the pelvis at multiple levels showing a highdensity ring structure containing air (a-c, arrowheads), located caudally in the left iliopsoas muscle. In the cranialmost image (a), the foreign body displaced, but did not actually penetrate the descending colon (long arrow). Gas can be seen dissecting between fibers of the iliacus muscle. At a more caudal level, the surrounding muscle demonstrated slightly lower attenuation, consistent with edema (b, c, arrows). A tiny amount of gas is seen in the medial aspect of the hip joint (c, long arrow), indicating that the joint capsule was penetrated. The foreign body extended craniocaudally approximately $13 \mathrm{~cm}$, with the caudal end of the foreign body at the level of the left hip joint (c). More caudally (d), gas tracked within the muscles of the left thigh, but no additional fragments of the foreign body were identified

area of extremely low attenuation in the left orbit of a boy who had been poked in the eye with a tree branch. The appearance and attenuation of the area suggested air, so a diagnosis of orbital emphysema was initially considered.

There are numerous reports of injury due to ingested wooden foreign bodies detected by CT. Strauss et al. [9] reported CT demonstration of jejunal perforation by an ingested toothpick. CT showed a linear high density pointing toward a fluid collection containing air. Peh et al. [10] described CT findings of a case of bamboo skewer perforation of the bowel. CT demonstrated a dense, forked foreign body within an extraluminal mass. A $2.5-\mathrm{cm}$ bamboo skewer surrounded by inflamed omentum was found at laparotomy.

Kadir et al. [5] soaked various types of wood for $24 \mathrm{~h}$ in water and found $\mathrm{CT}$ attenuation ranging from -276 to +27 EMI attenuation units ( -552 to $+54 \mathrm{HU})$. Heavy
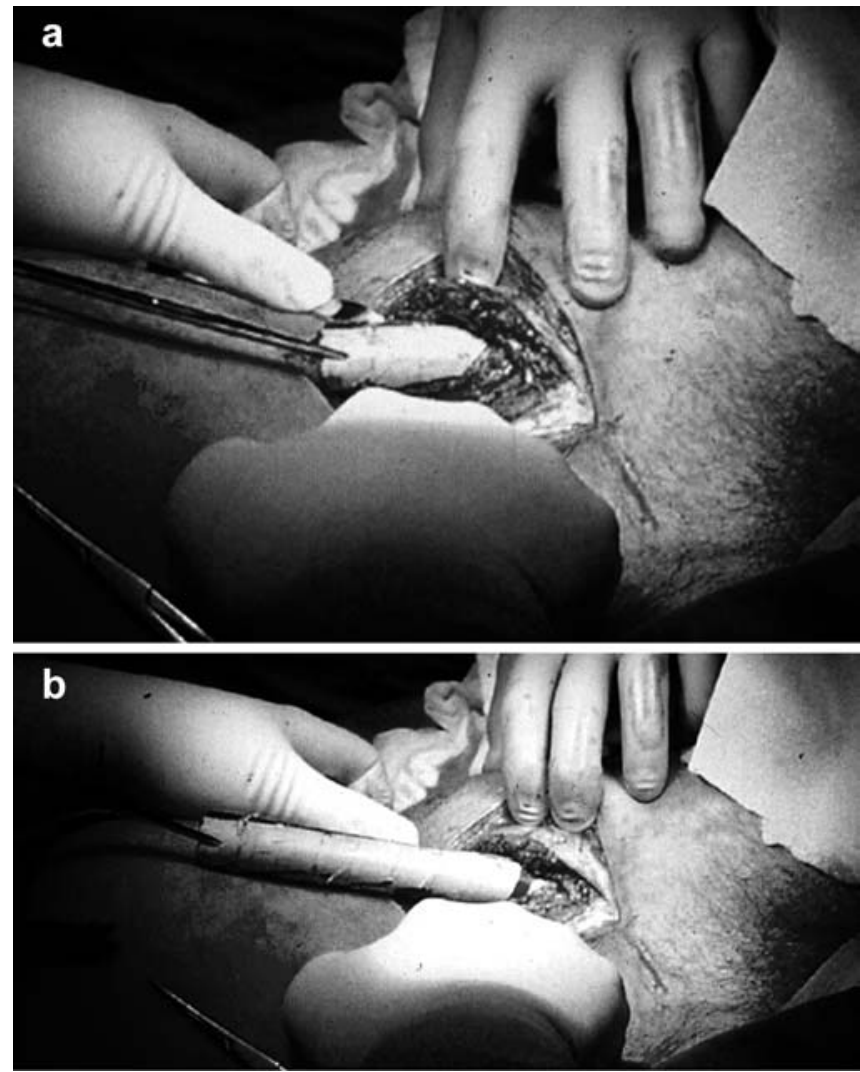

Fig. 4a, b. Intraoperative photographs. a Initial incision, exposing the taped-wrapped bamboo stick fragment. The entire $13-\mathrm{cm}$ stick (b) was extracted from the incision without complication 
woods showed substantially higher attenuation than that of water, whereas light woods, plywood, and particle boards showed attenuation similar to or lower than that of water and sometimes approximated that of air. The authors speculated that the low attenuation was due to trapped air.

In the present case, the location of the foreign body was mainly intramuscular, and the surrounding soft tissue provided good contrast. The CT attenuation value of the rim of the bamboo stick ranged from 127.5 to $156 \mathrm{HU}$ (average 143.3 HU), adjacent edematous muscle averaged $36 \mathrm{HU}$, and normal skeletal muscle averaged $67 \mathrm{HU}$.

In our case, the bamboo stick could have been identified on the trauma portable radiograph as a long rectangular gas lucency overlying the left pelvis, hip, and thigh. Prospectively, the radiologist was only aware that the patient had been injured skiing. Not suspecting such a large foreign body, the radiologist apparently dismissed the shadow as an artifact related to the backboard. This emphasizes three points: (1) the importance of an accurate history when foreign bodies are suspected, (2) the need to remove clothing and other extraneous material when possible, in order to assess more accurately for foreign bodies, and (3) the necessity for the radiologist to maintain a high index of suspicion in all trauma cases, and to diligently search the trauma film for any unexplained shadows.

In summary, this unique case of bamboo stick penetration from the posterior thigh to the anterior pelvis showed typical CT findings, demonstrating a hollow cylindrical structure of relatively high attenuation, with air within the lumen of the stick. Identification and evaluation by CT enabled the surgeon to plan the removal of the foreign body more effectively.

\section{References}

1. Hollerman JJ, Fackler ML, Coldwell DM, Ben-Menachem Y (1990) Gunshot wounds: 2. Radiology. AJR Am J Roentgenol 155:691-702

2. Bodene D, Quinn SF, Cochran CF (1988) Imaging foreign glass and wooden bodies of the extremities with CT and MR. J Comput Assist Tomogr 12:608-611

3. Uchino A, Kato A, Takase Y, Kudo S (1997) Intraorbital wooden and bamboo foreign bodies: CT. Neuroradiology $39: 213-215$

4. Greaney MJ (1994) Bamboo orbital foreign body mimicking air on computed tomography. Eye 8:713-714

5. Kadir S, Aronow S, Davis KR (1997) The use of computerized tomography in the detection of intra-orbital foreign bodies. Computerized Tomogr 1:151-156

6. Lindahl S (1987) Computed tomography of intraorbital foreign bodies. Acta Radiol 28:235-240

7. Roberts CF, Leehey PJ III (1992) Intraorbital wood foreign body mimicking air at CT. Radiology 185:507-508

8. Berger P, Kuhn JP, Kuhns LR (1980) Computed tomography and the occult tracheobronchial foreign body. Radiology 134:133-135

9. Strauss JE, Balthazar EJ, Naidich DP (1985) Jejunal perforation by a toothpick: CT demonstration. J Comput Assist Tomogr 9:812-814

10. Peh WC, Helpert C, Chan CW (1997) Bamboo skewer perforation of the bowel:computed tomography appearances. Australas Radiol 41:308-310

11. Nakata H, Egashira K, Nakamura K, Hayashi K, Mori M (1992) Bamboo foreign bodies in lung parenchyma: CT features. Clin Imaging 16:117-120

12. Kuhns LR, Borlaza GS, Siegel RS, Paramagul C, Berger PE (1979) An in vitro comparison of computed tomography, xeroradiography, and radiography in the detection of soft-tissue foreign bodies. Radiology 132:218-219

13. Horton LK, Jacobson JA, Powell A, Fessell DP, Hayes CW (2001) Sonography and radiography of soft-tissue foreign bodies. AJR Am J Roentgenol 176:1155-1159 